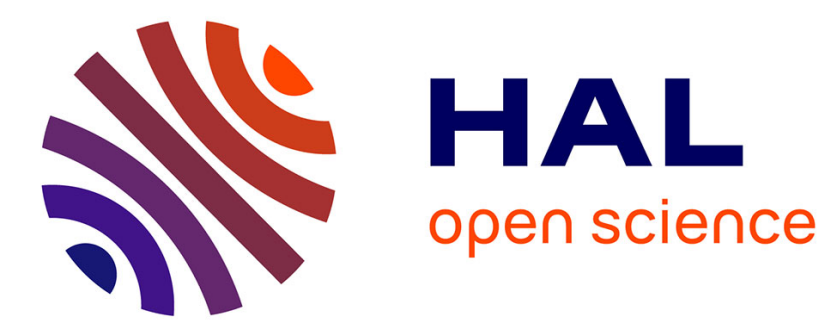

\title{
Controllability of Linear Systems on Lie Groups Philippe Jouan
}

\section{To cite this version:}

Philippe Jouan. Controllability of Linear Systems on Lie Groups. Journal of Dynamical and Control Systems, 2011, 17 (4), pp.591-616. hal-00541489

\section{HAL Id: hal-00541489 \\ https://hal.science/hal-00541489}

Submitted on 30 Nov 2010

HAL is a multi-disciplinary open access archive for the deposit and dissemination of scientific research documents, whether they are published or not. The documents may come from teaching and research institutions in France or abroad, or from public or private research centers.
L'archive ouverte pluridisciplinaire HAL, est destinée au dépôt et à la diffusion de documents scientifiques de niveau recherche, publiés ou non, émanant des établissements d'enseignement et de recherche français ou étrangers, des laboratoires publics ou privés. 


\title{
Controllability of Linear Systems with inner derivation on Lie Groups
}

\author{
Philippe JOUAN*
}

November 2, 2010

\begin{abstract}
A vector field on a connected Lie group is said to be linear if its flow is a one parameter group of automorphisms. A control-affine system is linear if the drift is linear and the controlled vector fields right invariant.

The controllability properties of such systems are studied, mainly in the case where the derivation of the group Lie algebra that can be associated to the linear vector field is inner.

After some general considerations controllability properties on semi simple, nilpotent and compact Lie groups are stated.

The paper ends by many examples.

Keywords: Lie groups; Linear systems; controllability; time optimal control.
\end{abstract}

AMS Subject Classification: 93B05; 22E15; 93B06; 93C10.

\section{Introduction}

The aim of this paper is to study the controllability properties of linear systems on Lie groups. By linear system is meant a controlled system

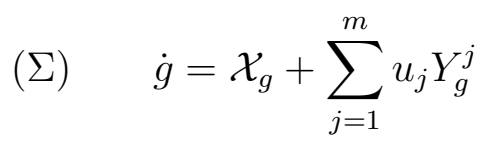

*Lab. R. Salem, CNRS UMR 6085, Université de Rouen, avenue de l'université BP 12, 76801 Saint-Étienne-du-Rouvray France. E-mail: Philippe.Jouan@univ-rouen.fr 
on a connected group Lie $G$ where $\mathcal{X}$ is a linear vector field, that is a vector field whose flow is a one parameter group of automorphisms, and the $Y_{j}$ 's are right invariant. Linear vector fields on Lie groups are nothing else than the so-called infinitesimal automorphisms in the Lie group literature (see for instance [3]). They were first considered in a control theory context by Markus, on matrix Lie groups ([12]), and then in the general case by Ayala and Tirao ([1]).

The motivation for dealing with such systems is twofold. On the one hand they are natural extensions of invariant systems on Lie groups. On the other hand they can be generalized to homogeneous spaces and appear as models for a wide class of systems, on account of the Equivalence Theorem of [7]. This equivalence was used in [9] to establish new controllability criterions on compact manifolds.

On Lie groups a few controllability properties of linear systems have already been proved ([1], [17], [4]):

1. On a compact and connected Lie group a linear system is controllable if and only it satisfies the rank condition. It was proved in [17].

2. A criterion of local controllability (the so-called ad-rank condition, see Section 3.4) was stated in [1] and [4].

3. If a linear system on a semisimple Lie group with finite center is controllable from the identity, then a certain invariant system, closely related to the linear one, is also controllable (see [17]).

A linear vector field acts on the Lie algebra $\mathfrak{g}$ of $G$, via the adjoint representation, as a derivation. A large part of this paper deals with the case where this derivation is inner. A right invariant system is then associated to the linear one in a natural way, different from the one of [17] (see Section 4.1). The controllability properties of those two systems are compared and in particular the controllability of the linear system is related to a time optimal problem for the invariant one.

This analysis is applied to semisimple Lie groups in Section 5.1. First of all it is stated in Theorem 2 that the invariant system is controllable as soon as the linear one is controllable from the identity, or to the identity. This statement is almost the same as the one of [17], the difference being due to the simpler way an invariant system is associated to $\Sigma$. Theorem 3 contains the following converse to Theorem 2 : 
Let $G$ be a semi-simple, connected Lie group with finite center. The adrank condition is assumed to hold. Then the linear system is controllable if and only if the invariant one is.

Together with the classical result of [5], this theorem furnishes controllability criterions on semisimple Lie groups.

Section 5.2 deals with nilpotent Lie groups. Theorem 4 asserts that: on a nilpotent connected Lie group, and whenever the derivation is inner, $\Sigma$ is controllable if and only if the algebra generated by $Y^{1}, \ldots, Y^{m}$ is equal to $\mathfrak{g}$. Some examples show that this theorem is no longer true on solvable Lie groups.

Finally we consider affine systems (Section 6). An affine vector field is the sum of a linear vector field and a right invariant one, and an affine system is obtained by replacing the drift of a linear system by an affine vector field. Both invariant and linear systems appear as particular cases of affine systems. Their properties are studied in Section 6, and then applied to compact Lie groups.

This allows to state Theorem 5 which generalizes the known results about invariant and linear systems: An affine control system on a compact and connected Lie group is controllable if and only if it satisfies the rank condition.

Section 7 is devoted to examples. Through these ones it is shown that the ad-rank condition is neither a necessary nor a sufficient controllability condition, and that a linear system can be controllable without being finite time controllable.

\section{Linear vector fields and linear systems}

In this section the definition of linear vector fields and some of their properties are recalled. More details can found in [7].

A vector field on a connected Lie group $G$ is said to be linear if its flow is a one parameter group of automorphisms. The following characterizations will be useful in the sequel.

Let $\mathcal{X}$ be a vector field on a connected Lie group $G$. The following conditions are equivalent:

1. $\mathcal{X}$ is linear;

2. $\mathcal{X}$ belongs to the normalizer of $\mathfrak{g}$ in the algebra of analytic vector fields 
of $G$, that is

$$
\forall Y \in \mathfrak{g} \quad[\mathcal{X}, Y] \in \mathfrak{g}
$$

and verifies $\mathcal{X}(e)=0$;

3. $\mathcal{X}$ verifies

$$
\forall g, g^{\prime} \in G \quad \mathcal{X}_{g g^{\prime}}=T L_{g} \cdot \mathcal{X}_{g^{\prime}}+T R_{g^{\prime}} \cdot \mathcal{X}_{g}
$$

On account of the second item the derivation $D=-\operatorname{ad}(\mathcal{X})$ of the Lie algebra $\mathfrak{g}$ of $G$ is associated to the linear vector field $\mathcal{X}$.

In the case where this derivation is inner, that is $D=-\operatorname{ad}(X)$ for some right invariant vector field $X$ on $G$, the linear vector field splits into $\mathcal{X}=$ $X+\mathcal{I}_{*} X$, where $\mathcal{I}$ stands for the diffeomorphism $g \in G \longmapsto \mathcal{I}(g)=g^{-1}$. Thus $\mathcal{X}$ is the sum of the right invariant vector field $X$ and the left invariant one $\mathcal{I}_{*} X$.

The flow of a linear vector field $\mathcal{X}$ will be denoted by $\left(\varphi_{t}\right)_{t \in \mathbb{R}}$. In the inner derivation case it is given by

$$
\varphi_{t}(g)=\exp (t X) g \exp (-t X)
$$

Definition $1 A$ linear system on a connected Lie group $G$ is a controlled system

$$
\text { ( } \Sigma) \quad \dot{g}=\mathcal{X}_{g}+\sum_{j=1}^{m} u_{j} Y_{g}^{j}
$$

where $\mathcal{X}$ is a linear vector field and the $Y^{j}$ 's are right invariant ones. The control $u=\left(u_{1}, \ldots, u_{m}\right)$ takes its values in $\mathbb{R}^{m}$.

The set $\mathbf{U}$ of admissible inputs is a subset of $L_{l o c}^{\infty}\left(\left[0,+\infty\left[, \mathbb{R}^{m}\right)\right.\right.$ which contains the piecewise constant functions and is stable for concatenation, that is if $\omega$ and $\nu$ belong to $\mathbf{U}$, then the function $w$ defined by

$$
w(t)= \begin{cases}\omega(t) & t \in[0, T[ \\ \nu(t-T) & t \in[T,+\infty[\end{cases}
$$

belongs as well to $\mathbf{U}$.

Such an input being given $g_{u}(t)$ (or briefly $g(t)$ when no confusion can arrive) stands for the trajectory of $\Sigma$ which verifies $g_{u}(0)=g$.

Finally, let us recall the definition of the so-called zero-time ideal. Let $\dot{x}=$ $f(x)+\sum_{j=1}^{m} u_{j} g^{j}(x)$ be a $\mathcal{C}^{\infty}$ control-affine system on a connected manifold, and let $\mathcal{L}$ be the Lie algebra of $\mathcal{C}^{\infty}$ vector fields generated by $f, g_{1}, \ldots, g_{m}$. 
The zero-time ideal $\mathcal{L}_{0}$ of the system is the smallest ideal of $\mathcal{L}$ that contains $g_{1}, \ldots, g_{m}$. It satisfies the equality

$$
\mathcal{L}=\mathbb{R} f+\mathcal{L}_{0}
$$

\section{General properties}

\subsection{The reachable sets}

Proposition 1, that can be found in [4], is fundamental. Its proof is straightforward.

Proposition 1 ([4]) The input u being given, let us denote by e(t) the trajectory of $\Sigma$ issued from the identity e of $G$. For the initial condition $g$ the trajectory is then

$$
g(t)=e(t) \varphi_{t}(g)
$$

Remark. If the vector fields $Y^{j}$ were left invariant we would have $g(t)=$ $\varphi_{t}(g) e(t)$.

Notations. We denote by $\mathcal{A}(g, t)=\left\{g_{u}(t) ; u \in L^{\infty}[0, t]\right\}(\operatorname{resp} . \mathcal{A}(g, \leq t))$ (resp. $\mathcal{A}(g)$ ) the reachable set from $g$ in time $t$ (resp. in time less than or equal to $t$ ) (resp. in any time). In particular the reachable sets from the identity $e$ are denoted by

$$
\mathcal{A}_{t}=\mathcal{A}(e, t) \quad \text { and } \quad \mathcal{A}=\mathcal{A}(e) .
$$

These sets are related by the following equalities and inclusions:

Proposition 2 1. $\forall t \geq 0 \quad \mathcal{A}(e, \leq t)=\mathcal{A}(e, t)=\mathcal{A}_{t}$.

2. $\forall 0 \leq s \leq t \quad \mathcal{A}_{s} \subset \mathcal{A}_{t}$.

3. $\forall g \in G \quad \mathcal{A}(g, t)=\mathcal{A}_{t} \varphi_{t}(g)$.

4. $\forall s, t \geq 0 \quad \mathcal{A}_{t+s}=\mathcal{A}_{t} \varphi_{t}\left(\mathcal{A}_{s}\right)=\mathcal{A}_{s} \varphi_{s}\left(\mathcal{A}_{t}\right)$.

Proof. The identity being an equilibrium of $\mathcal{X}$, the first two items are standard and can be found for instance in [19].

The last two are consequences of Proposition 1. 


\subsection{The system Lie algebra and the rank condition}

Let $\mathfrak{h}$ be the subalgebra of $\mathfrak{g}$ generated by $\left\{Y^{1}, \ldots, Y^{m}\right\}$, and let us denote by $D \mathfrak{h}$ the smallest $D$-invariant subspace of $\mathfrak{g}$ that contains $\mathfrak{h}$, where $D$ is the derivation associated to $\mathcal{X}$ :

$$
D \mathfrak{h}=\operatorname{Sp}\left\{D^{k} Y ; Y \in \mathfrak{h} \text { and } k \in \mathbb{N}\right\} .
$$

Let $\mathcal{L} \mathcal{A}(D \mathfrak{h})$ stand for the $\mathfrak{g}$ subalgebra generated by $D \mathfrak{h}$.

Proposition 3 The subalgebra $\mathcal{L} \mathcal{A}(D \mathfrak{h})$ is D-invariant. It is therefore equal to the zero-time ideal $\mathcal{L}_{0}$, and the system Lie algebra $\mathcal{L}$ to

$$
\mathbb{R} \mathcal{X} \oplus \mathcal{L} \mathcal{A}(D \mathfrak{h})=\mathbb{R} \mathcal{X} \oplus \mathcal{L}_{0}
$$

The rank condition is satisfied by $\Sigma$ if and only if $\mathcal{L}_{0}=\mathfrak{g}$.

Proof.

First of all the algebra $\mathcal{L} \mathcal{A}(D \mathfrak{h})$ is $D$-invariant. Indeed the elements of $D \mathfrak{h}$ are linear combinations of $D^{k} Y$ where $Y \in \mathfrak{h}$ and $k \geq 0$, and

$$
\forall Y, Z \in \mathfrak{h} \quad D\left[D^{k} Y, D^{l} Z\right]=\left[D^{k+1} Y, D^{l} Z\right]+\left[D^{k} Y, D^{l+1} Z\right] \in \mathcal{L} \mathcal{A}(D \mathfrak{h}) .
$$

The zero-time ideal being the ideal of $\mathcal{L}$ generated by $Y^{1}, \ldots, Y^{m}$, it is clearly equal to $\mathcal{L} \mathcal{A}(D \mathfrak{h})$. The system Lie algebra is consequently $\mathcal{L}=$ $\mathbb{R} \mathcal{X} \oplus \mathcal{L} \mathcal{A}(D \mathfrak{h})$. As $\mathcal{X}(e)=0$, the rank at the point $e$ is maximum if and only if $\mathcal{L} \mathcal{A}(D \mathfrak{h})=\mathfrak{g}$. In that case the rank condition is everywhere satisfied.

\subsection{The Lie saturate}

The Lie saturate $\mathcal{L S}(\Sigma)$ of $\Sigma$ (resp. the strong Lie saturate $\mathcal{L} \mathcal{S} \mathcal{S}(\Sigma)$ of $\Sigma$ ) is the set of vector fields $f$ belonging to the system Lie algebra $\mathcal{L}$ and whose flow $\left(\phi_{t}\right)_{t \in \mathbb{R}}$ satisfies

$$
\forall g \in G, \forall t \geq 0 \quad \phi_{t}(g) \in \overline{\mathcal{A}(g)} \quad\left(\text { resp. } \phi_{t}(g) \in \overline{\mathcal{A}(g, \leq t)}\right)
$$

as soon as $\phi_{t}(g)$ is defined. The notion of Lie saturate as well as the proof of Proposition 4 can be found in [11]. 
Proposition 4 The algebra $\mathfrak{h}$ is included in $\mathcal{L} \mathcal{S} \mathcal{S}(\Sigma)$, so that $\Sigma$ can be enlarged to the system

$$
(\widetilde{\Sigma}) \quad \dot{g}=\mathcal{X}_{g}+\sum_{j=1}^{p} u_{j} \widetilde{Y}_{g}^{j},
$$

where $\widetilde{Y}^{1}, \ldots, \widetilde{Y}^{p}$ is a basis of $\mathfrak{h}$, without modifying the sets $\overline{\mathcal{A}(g, \leq t)}$.

Proof. Cf [11].

Assume now that the system satisfies the rank condition. Let $H$ be the connected Lie subgroup of $G$ whose Lie algebra is $\mathfrak{h}$, and let us denote by $\overline{\mathfrak{h}}$ the Lie algebra of the closure $\bar{H}$ of $H$ in $G$.

Proposition 5 If $\Sigma$ satisfies the rank condition, then the algebra $\overline{\mathfrak{h}}$ is contained in $\mathcal{L S S}(\Sigma)$.

Proof.

According to Proposition $4, H g \subset \overline{\mathcal{A}(g, \leq t)}$ for every $g \in G$ and every $t>0$. Therefore $\bar{H} g$ is as well included in $\overline{\mathcal{A}(g, \leq t)}$. The rank condition being satisfied, $\overline{\mathfrak{h}} \subset \mathfrak{g}$ is contained in the Lie algebra $\mathcal{L}$ of $\Sigma$, and $\overline{\mathfrak{h}} \subset \mathcal{L} \mathcal{S} \mathcal{S}(\Sigma)$.

The interest of this second enlargement is rather theoretical since it may be hard to decide if the subgroup $H$ is closed in $G$.

\subsection{Local controllability and the ad-rank condition}

It is well known that a system is locally controllable at an equilibrium point as soon as the linearized system is controllable (see [13] for instance). In this assertion "locally controllable" at a point $g$ means that the set $\mathcal{A}(g, t)$ is a neighbourhood of $g$ for all $t>0$.

In view of Proposition 4 it may be more interesting to consider the linearization at the identity of the enlarged system $\widetilde{\Sigma}$ rather than the one of $\Sigma$.

Example (See Section 7). The linearized system at $e$ of System $L_{3}$ on the Heisenberg group is not controllable. However the controlled vector fields of $L_{3}$ generate the Lie algebra $\mathfrak{g}$ and the linearization of the enlarged system is controllable.

These remarks lead to define a stronger rank condition, called "ad-rank condition" in [1]. 
Definition 2 System $(\Sigma)$ is said to satisfy the ad-rank condition if $D \mathfrak{h}=$ $\mathfrak{g}$.

Proposition 6 (See [1]) If the ad-rank condition is satisfied then for all $t>0$ the reachable set $\mathcal{A}_{t}$ is a neighbourhood of $e$.

In [4] the weaker result that under the ad-rank condition the attainable set $\mathcal{A}$ is a neighbourhood of $e$ is stated, using the theory of Lie semigroups. The proof presented here is novel, and is actually an easy consequence of the linearization principle.

Proof.

It is proved in [7] that the tangent mapping at $e$ of the flow of $\mathcal{X}$ is $T_{e} \varphi_{t}=e^{t D}$. According to this equality the linearized vector field of $\mathcal{X}$ at $e$ is the endomorphism $D$ of $T_{e} G \simeq \mathfrak{g}$.

Thus the linearization of $\widetilde{\Sigma}$ at $e$ can be written in $T_{e} G$

$$
\dot{Z}=D Z+\sum_{j=1}^{m} u_{j} \widetilde{Y}^{j}(e)
$$

where $\left(\widetilde{Y}^{1}, \ldots, \widetilde{Y}^{m}\right)$ is a basis of $\mathfrak{h}$. The ad-rank condition is therefore equivalent to the Kalman rank condition for this linear system.

Important remark. The ad-rank condition is not a sufficient condition of controllability. It is for instance satisfied by Example 2 in Section 7.3 although the system is not controllable on $S L_{2}$, not even from the identity.

\subsection{Groups and semigroups}

In this section we consider the polysystem $\{\mathcal{X}\} \cup \mathfrak{h}$ instead of $\Sigma$. According to Proposition 4 and to enlargement techniques ([11]), the closure of the sets $\mathcal{A}_{t}$ is not modified. The set of admissible inputs is restricted to the piecewise constant ones, and the results are stated within this framework.

Lemma 1 For all $t>0$ the set $\mathcal{A}_{t}$ is equal to

$$
\begin{aligned}
& \left\{\varphi_{t_{1}}\left(h_{1}\right) \varphi_{t_{2}}\left(h_{2}\right) \ldots \varphi_{t_{n}}\left(h_{n}\right) ;\right. \\
& \left.n \in \mathbb{N}, \quad h_{1}, \ldots, h_{n} \in H, \quad 0 \leq t_{1} \leq t_{2} \leq \cdots \leq t_{n}<t\right\},
\end{aligned}
$$

where $H$ is the connected subgroup of $G$ whose Lie algebra is $\mathfrak{h}$. 
Proof. For the system under consideration it is clear that $H \subset \mathcal{A}_{s}$ for all $s>0$.

The proposition is due to the fact that a point $g$ of the form

$$
g=\varphi_{t_{1}}\left(h_{1}\right) \varphi_{t_{2}}\left(h_{2}\right) \ldots \varphi_{t_{n}}\left(h_{n}\right) \text {, with } 0 \leq t_{1} \leq t_{2} \leq \cdots \leq t_{n},
$$

can be written

$$
g=\varphi_{t_{1}}\left(h_{1} \varphi_{t_{2}-t_{1}}\left(h_{2} \ldots\left(h_{n-1} \varphi_{t_{n}-t_{n-1}}\left(h_{n}\right)\right)\right)\right) \text {, with } t_{i+1}-t_{i} \geq 0 .
$$

Remark. In the previous statement the inequality $t_{n}<t$ always holds. The points $h_{i}$ are indeed reached in an arbitrary but positive time (except in the particular case where all the $h_{i}$ 's are equal to $e$ ).

In the paper [1] it is conjectured that the set $\mathcal{A}$ is not a semigroup in general. We show the stronger result that $\mathcal{A}$ cannot be a semigroup without being a group.

Let $S$ stand for the semigroup generated by $\mathcal{A}$. It is clear that the elements of $S$ can be characterized in a similar way as the ones of $\mathcal{A}$ in Lemma 1, with the condition $t_{1}, t_{2}, \ldots, t_{n} \geq 0$ instead of $0 \leq t_{1} \leq t_{2} \leq \cdots \leq t_{n}$. In order to show that $S$ is a group it is enough to prove that $g \in S \Longrightarrow g^{-1} \in S$. Let

$$
g=\varphi_{t_{1}}\left(h_{1}\right) \varphi_{t_{2}}\left(h_{2}\right) \ldots \varphi_{t_{n}}\left(h_{n}\right) \in S, \text { with } t_{1}, t_{2}, \ldots, t_{n} \geq 0 \text {. }
$$

Then

$$
g^{-1}=\varphi_{t_{n}}\left(h_{n}^{-1}\right) \ldots \varphi_{t_{2}}\left(h_{2}^{-1}\right) \varphi_{t_{1}}\left(h_{1}^{-1}\right)
$$

belongs as well to $S$. Moreover if $\Sigma$ satisfies the rank condition, then $S$ is a subgroup of $G$ that contains a nonempty open set, hence $S=G$. We have proved:

Proposition 7 The reachable set $\mathcal{A}$ is a group if and only if it is a semigroup. In particular if the rank condition is satisfied then

$$
\mathcal{A}=G \Longleftrightarrow \mathcal{A} \text { is a semigroup. }
$$

The following corollary can be easily deduced from Proposition 7. However it is of limited interest because of the difficulty to check its conditions. 
Corollary 1 If the rank condition is satisfied, and if there exists $T>0$ such that $\mathcal{A}_{T}=\mathcal{A}$, then $\Sigma$ is controllable at time $T$.

Moreover the second condition holds as soon as there exist $T>0$ and $\epsilon>0$ such that $\mathcal{A}_{T}=\mathcal{A}_{T+\epsilon}$.

Proof. As $S=G$, any $g \in G$ can be written

$$
g=\varphi_{t_{1}}\left(h_{1}\right) \varphi_{t_{2}}\left(h_{2}\right) \ldots \varphi_{t_{n}}\left(h_{n}\right),
$$

with $h_{i} \in H$ and $t_{i} \geq 0$. Denoting $g_{i}=\varphi_{t_{i}}\left(h_{i}\right) \in \mathcal{A}=\mathcal{A}_{T}$ we get

$$
\begin{aligned}
\varphi_{T}(g) & =\varphi_{T+t_{1}}\left(h_{1}\right) \varphi_{T+t_{2}}\left(h_{2}\right) \ldots \varphi_{T+t_{n}}\left(h_{n}\right) \\
& =\varphi_{T}\left(g_{1}\right) \varphi_{T}\left(g_{2}\right) \ldots \varphi_{T}\left(g_{n}\right) .
\end{aligned}
$$

But $\varphi_{T}\left(g_{1}\right) \in \mathcal{A}_{2 T}=\mathcal{A}_{T}$. Hence $\varphi_{T}\left(g_{1}\right) \varphi_{T}\left(g_{2}\right) \in \mathcal{A}_{T} \varphi_{T}\left(\mathcal{A}_{T}\right)=\mathcal{A}_{2 T}=\mathcal{A}_{T}$, and by an obvious induction $\varphi_{T}(g) \in \mathcal{A}_{T}$. This shows that $G=\varphi_{T}(G) \subset \mathcal{A}_{T}$.

The proof of the second assertion is standard (see [19]).

\section{The inner derivation case}

In this section the derivation $\operatorname{ad}(\mathcal{X})$ is assumed to be inner. A right invariant system is associated in a natural way to $\Sigma$ and its controllability is related to the one of the linear system.

\subsection{The associated invariant system}

Since the derivation $D=a d(\mathcal{X})$ is inner, the vector field $\mathcal{X}$ can be decomposed into

$$
\mathcal{X}=X+\mathcal{I}_{*} X
$$

where $X$ is a right invariant vector field that satisfies $a d(X)=a d(\mathcal{X})$ (see Section 2).

It is thus natural to define on $G$ the right invariant system:

$$
\left(\Sigma_{I}\right) \quad \dot{g}=X_{g}+\sum_{j=1}^{m} u_{j} Y_{g}^{j}
$$

In order to avoid confusion the linear system will be denoted by $\Sigma_{L}$ in this section. 
Proposition 8 Let $t \longmapsto u(t)$ be an admissible input. The absolutely continuous curve $t \longmapsto g_{L}(t)$ is solution of the linear system $\Sigma_{L}$ for this input if and only if the curve $t \longmapsto g_{L}(t) \exp (t X)$ is solution of the right invariant system $\Sigma_{I}$.

Proof. If $t \longmapsto g_{L}(t)$ is solution of the linear system, then for almost every $t$ :

$$
\begin{aligned}
\frac{d}{d t} g_{L}(t) \exp (t X)= & T L_{g_{L}(t)} \cdot X_{\exp (t X)}+T R_{\exp (t X) \cdot\left(\mathcal{X}_{g_{L}(t)}+\sum_{j=1}^{m} u_{j}(t) Y_{g_{L}(t)}^{j}\right)} \\
=T L_{g_{L}(t)} \cdot X_{\exp (t X)} & +T R_{\exp (t X) \cdot\left(X_{g_{L}(t)}+\left(\mathcal{I}_{*} X\right)_{g_{L}(t)}\right)}+\sum_{j=1}^{m} u_{j}(t) Y_{g_{L}(t) \exp (t X)}^{j} \\
= & T L_{g_{L}(t)} T R_{\exp (t X)} \cdot X_{e}+X_{g_{L}(t) \exp (t X)}+T R_{\exp (t X)}\left(\mathcal{I}_{*} X\right)_{g_{L}(t)} \\
& +\sum_{j=1}^{m} u_{j}(t) Y_{g(t) \exp (t X)}^{j} \\
= & X_{g_{L}(t) \exp (t X)}+\sum_{j=1}^{m} u_{j}(t) Y_{g_{L}(t) \exp (t X)}^{j}
\end{aligned}
$$

since

$$
T R_{\exp (t X)}\left(\mathcal{I}_{*} X\right)_{g_{L}(t)}=-T R_{\exp (t X)} T L_{g_{L}(t)} \cdot X_{e}=-T L_{g_{L}(t)} T R_{\exp (t X)} \cdot X_{e} .
$$

The converse is similar.

We will use the symbol $\mathcal{S}$ in place of $\mathcal{A}$ to denote the reachable sets of the invariant system. A straightforward consequence of Proposition 8 is that the set $\mathcal{S}_{t}$ of reachable points from $e$ in time $t$ verifies

$$
\forall t \geq 0 \quad \mathcal{S}_{t}=\mathcal{A}_{t} \exp (t X) .
$$

The relation between the reachable sets in time less than or equal to $T>0$ is a bit more complicated:

$$
\mathcal{S}_{\leq T}=\bigcup_{0 \leq t \leq T} \mathcal{A}_{t} \exp (t X)
$$

It can also be noticed that both systems have the same zero-time ideal $\mathcal{L}_{0}$. Indeed since $D=\operatorname{ad}(\mathcal{X})=\operatorname{ad}(X)$ this ideal is in both cases the smallest 
$D$-invariant subalgebra of $\mathfrak{g}$ that contains $Y^{1}, \ldots, Y^{m}$. Consequently the Lie algebras of the systems are

$$
\mathcal{L}\left(\Sigma_{L}\right)=\mathbb{R} \mathcal{X} \oplus \mathcal{L}_{0} \quad \text { and } \quad \mathcal{L}\left(\Sigma_{I}\right)=\mathbb{R} X \oplus \mathcal{L}_{0}
$$

If the linear system satisfies the rank condition, then $\mathcal{L}_{0}=\mathfrak{g}$ and the invariant one verifies $\mathcal{L}\left(\Sigma_{I}\right)=\mathcal{L}_{0}=\mathfrak{g}$.

\subsection{Finite time controllability}

The remarks made in the previous subsection about the Lie algebras of $\Sigma_{L}$ and $\Sigma_{I}$ have consequences on finite time controllability.

Let us recall that a control system is said to be finite time controllable (resp. exact time controllable) if there exists $T>0$ such that any point $x$ can be steered to any point $y$ in time less than or equal to $T$ (resp. in time $T)$.

It is shown in [8] that a Lie determined and finite time controllable system is exact time controllable if and only if its zero-time ideal satisfies the rank condition. For a linear system finite time and exact time controllability are therefore always equivalent. This equivalence is not true in general for invariant systems, but it holds whenever the associated linear system satisfies the rank condition.

Proposition 9 Let $T>0$. The following assertions are equivalent:

1. $\Sigma_{L}$ is controllable in time less than or equal to $T$;

2. $\Sigma_{L}$ is controllable in time $T$;

3. $\Sigma_{L}$ is controllable from the identity in time $T$;

4. $\Sigma_{I}$ is controllable from the identity in time $T$;

5. $\Sigma_{I}$ is controllable in time $T$;

These conditions are moreover equivalent to: $\Sigma_{I}$ is finite time controllable and $\Sigma_{L}$ satisfies the rank condition.

Proof.

According to the following facts: 
1. $\forall t \geq 0, \quad \mathcal{S}_{t}=\mathcal{A}_{t} \exp (t X)$;

2. $\forall g \in G, \forall t \geq 0, \mathcal{S}(g, t)=\mathcal{S}_{t} g$;

3. $\forall g \in G, \forall t \geq 0, \mathcal{A}(g, t)=\mathcal{A}_{t} \varphi_{t}(g)=\mathcal{A}(e, \leq t) \varphi_{t}(g) ;$

the five conditions are equivalent to $\mathcal{A}_{T}=G$.

\section{Remarks.}

1. A linear system on a vector space cannot be controllable without being exact time controllable. That property is no longer true in the general case. An example of a controllable but not finite time controllable on the group $A f f_{+}(2)$ of affine transformations of the real line is exhibited in Section 7.2. The associated invariant system is not controllable.

2. In particular Proposition 9 applies to compact groups. However the controllability results on such groups can be extended to affine systems and for this reason are postponed to Section 6.1.

\subsection{Time optimal control of the invariant system}

In this subsection the invariant system is assumed to be controllable. Under this assumption, the controllability of the linear one is shown to be equivalent to a time optimal problem. It will be said that the the time $t>0$ is not optimal for $\exp (t X)$ if the trajectory $\tau \longmapsto \exp (\tau X)$ does not minimize the time necessary for the invariant system to steer the point $e$ to the point $\exp (t X)$.

Theorem 1 We assume the rank condition $\mathcal{L}_{0}=\mathfrak{g}$ to be satisfied and the right invariant system to be controllable. The following assertions are equivalent:

(i) there exists a time $t>0$ which is not optimal for $\exp (t X)$;

(ii) $\exp (\mathbb{R} X) \subset \mathcal{A}$;

(iii) The linear system is controllable.

These conditions are satisfied, and the linear system is controllable, as soon as the set $\mathcal{A}_{t}$ is a neighbourhood of e for some $t>0$, in particular whenever the ad-rank condition holds. 
Proof.

1. Let us first remark that the set $\{\tau \in \mathbb{R} ; \exp (\tau X) \in \mathcal{A}\}$ is a semigroup. Indeed if $\exp \left(\tau_{1} X\right) \in \mathcal{A}_{t_{1}}$ and $\exp \left(\tau_{2} X\right) \in \mathcal{A}_{t_{2}}$, then

$$
\exp \left(\tau_{1} X\right) \exp \left(\tau_{2} X\right)=\exp \left(\tau_{1} X\right) \varphi_{t_{1}}\left(\exp \left(\tau_{2} X\right)\right) \in \mathcal{A}_{\tau_{1}+\tau_{2}}
$$

because $\varphi_{t}(g)=\exp (t X) g \exp (-t X)$ (see Section 2). On the other hand $\Sigma_{I}$ being controllable and $\mathcal{L}_{0}$ equal to $\mathcal{L}$, there exists $t>0$ such that $\mathcal{S}_{t}$ is a neighbourhood of $e$ (see [8]). Therefore $\mathcal{S}_{t} \exp (-t X) \cap$ $\exp (\mathbb{R} X)$, which is included in $\mathcal{A}_{t}$, contains a neighbourhood (in the set $\exp (\mathbb{R} X))$ of $\exp (-t X)$. Together with the semigroup property, this gives:

$$
\exp (\mathbb{R} X) \subset \mathcal{A} \Longleftrightarrow \exists \tau>0 \text { such that } \exp (\tau X) \in \mathcal{A} \text {. }
$$

2. $(i) \Longleftrightarrow(i i)$

If $t>0$ is not optimal for $\exp (t X)$, then there exists $\tau$, with $0<$ $\tau<t$, such that $\exp (t X) \in \mathcal{S}_{\tau}$. Consequently $\exp ((t-\tau) X) \in \mathcal{A}$ and $\exp (\mathbb{R} X) \subset \mathcal{A}$.

Conversely if $\exp (\mathbb{R} X) \subset \mathcal{A}$, let us choose arbitrarily $\tau>0$. There exists $t>0$ such that $\exp (\tau X) \in \mathcal{A}_{t}$, hence such that $\exp ((t+\tau) X) \in$ $\mathcal{S}_{t}$. This shows that the time $t+\tau$ is not optimal for $\exp ((t+\tau) X)$.

3. $(i i) \Longrightarrow(i i i)$

Let us first show that the linear system is controllable from $e$.

Let $g \in G$. There exists $t \geq 0$ such that $g \in \mathcal{S}_{t}$, hence such that $g \exp (-t X) \in \mathcal{A}_{t}$. By assumption there exists $s>0$ such that $\exp (t X)$ belongs to $\mathcal{A}_{s}$, and thus:

$$
g=g \exp (-t X) \varphi_{t}(\exp (t X)) \in \mathcal{A}_{t} \varphi_{t}\left(\mathcal{A}_{s}\right)=\mathcal{A}_{t+s}
$$

Let us now show that $\Sigma_{L}$ is controllable to $e$. We have to prove that $e \in \mathcal{A}(g)$ for all $g \in G$.

As there exists $t \geq 0$ such that $g^{-1} \in \mathcal{S}_{t}$, or in an equivalent way such that $g^{-1} \exp (-t X) \in \mathcal{A}_{t}$, we have

$$
\begin{aligned}
\exp (-t X) & =g^{-1} \exp (-t X) \exp (t X) g \exp (-t X) \\
& =g^{-1} \exp (-t X) \varphi_{t}(g) \\
& \in \mathcal{A}(g, t)
\end{aligned}
$$


By assumption there exists $s>0$ such that $\exp (t X) \in \mathcal{A}_{s}$, and we obtain

$$
e=\exp (t X) \varphi_{s}(\exp (-t X)) \in \mathcal{A}(\exp (-t X), s) .
$$

This shows that $e \in \mathcal{A}(g)$.

$($ iii $) \Longrightarrow($ ii $)$ is obvious.

4. If the set $\mathcal{A}_{t}$ is a neighbourhood of $e$ for some $t>0$, then $\exp (\mathbb{R} X)$ is included in $\mathcal{A}$, and according to Condition ( $i i)$ the linear system is controllable.

\section{Application to some classes of Lie groups}

\subsection{Semisimple Lie groups}

Before applying Theorem 1 to semisimple groups, let us state Theorem 2 which is a reformulation of the main result of [17]. The idea of the proof, based on the notion of reversibility of semigroups, is also due to [17]. However the proof given here is simpler than the original one, thanks to the way we associate an invariant system to the linear one.

Theorem 2 ([17]) The group $G$ is assumed to be semisimple, connected, with finite center. Then the invariant system is controllable as soon as the linear one is controllable from the identity, or to the identity.

Proof.

Let $S$ be a generating subsemigroup of a group $G$. It is said to be left reversible (resp. right reversible) if $S S^{-1}=G$ (resp. $S^{-1} S=G$ ). The following statement can be found in [18]:

Let $G$ be a connected and semisimple Lie group with finite center. The only subsemigroup of $G$ with nonempty interior which is left or right reversible is $G$.

The set $\mathcal{S}$ of reachable points from $e$ for $\Sigma_{I}$ is a semigroup and, as the rank condition is satisfied, its interior is not empty. It is therefore sufficient to show that it is left, or right, reversible. 
1. Let us prove that $\Sigma_{L}$ controllable from $e$ implies $\mathcal{S}$ left reversible. Let $g \in G$. There exists $t \geq 0$ such that $g \in \mathcal{A}_{t}=\mathcal{S}_{t} \exp (-t X)$. This shows that $\mathcal{S} \exp \left(-\mathbb{R}_{+} X\right)=G$ and, as $\exp \left(\mathbb{R}_{+} X\right) \subset \mathcal{S}$, that $\mathcal{S} \mathcal{S}^{-1}=G$.

2. Let us prove that $\Sigma_{L}$ controllable to $e$ implies $\mathcal{S}$ right reversible. Let $g \in$ $G$. There exists $t \geq 0$ such that $e \in \mathcal{A}\left(g^{-1}, t\right)$, hence there exists $x \in \mathcal{A}_{t}$ such that $e=x \varphi_{t}\left(g^{-1}\right)$. Consequently $\varphi_{t}(g)=\exp (t X) g \exp (-t X)=$ $x \in \mathcal{A}_{t}$ and $\exp (t X) g \in \mathcal{S}_{t}$. Finally $\exp \left(-\mathbb{R}_{+} X\right) \mathcal{S}=G$ and $\mathcal{S}$ is right reversible.

Remark. Theorem 2 is no longer true whenever the center of $G$ is infinite. A counter-example is exhibited in the last section of [16].

The following corollary is a straightforward consequence of Theorems 1 and 2 .

Corollary 2 The group $G$ is assumed to be semisimple, connected and its center to be finite.

If the linear system $\Sigma_{L}$ is controllable from the identity $e$ then it is controllable.

Proof. According to Theorem 2 the invariant system is controllable. Since $\mathcal{A}=G$ Theorem 1 applies.

Remark. We do not know if this result holds on more general Lie groups.

In the case where the ad-rank condition is satisfied the controllability of $\Sigma_{L}$ and $\Sigma_{I}$ are equivalent. Moreover the sufficient conditions established by Rachida El Assoudi, Jean-Paul Gauthier and Ivan Kupka in [5] are valid for both systems. These conditions make appeal to the rather technical hypothesis of [5], that are detailed in Appendix 8.1.

Theorem 3 Let $G$ be a semi-simple, connected Lie group with finite center. The ad-rank condition is assumed to hold.

Then the linear system is controllable if and only if the invariant one is.

In particular both systems are controllable whenever the following conditions hold:

1. The algebra $\mathfrak{h}$ contains a strongly regular element $Y$. 
2. Let $X=X_{0}+\sum_{a \in S p(Y)} X_{a}$ be the decomposition of $X$ along the eigen spaces of $a d_{\mathbb{C}}(Y)$. Then $X_{a} \neq 0$ as soon as the eigenvalue $a \in S p(Y)$ is minimal or maximal.

3. If $a \in S p(Y)$ is maximal, if its real part $r=\Re(a)$ is a non zero eigenvalue of a $d_{\mathbb{C}}(Y)$, and if $\mathfrak{g}_{r}$ and $\mathfrak{g}_{a}$ belong to the same simple ideal of $\mathfrak{g}_{\mathbb{C}}$, then the trace of ad $\left(X_{r}\right) \circ$ ad $\left(X_{-r}\right)$ is negative.

Remark. In a semi-simple Lie algebra $\mathfrak{g}$ the set of pairs $\left(Y^{1}, Y^{2}\right)$ that generate $\mathfrak{g}$ is open and dense in $\mathfrak{g} \times \mathfrak{g}$. Both systems $\Sigma_{I}$ and $\Sigma_{L}$ are therefore generically controllable as soon as $m \geq 2$.

\subsection{Nilpotent Lie groups}

The subalgebra of $\mathfrak{g}$ generated by the fields $Y^{j}, j=1, \ldots, m$ is, as previously, denoted by $\mathfrak{h}$.

Theorem 4 The group $G$ is assumed to be nilpotent, and the derivation associated to $\mathcal{X}$ to be inner. Then $\Sigma$ is controllable if and only if $\mathfrak{h}=\mathfrak{g}$. In that case it is controllable in exactly $T$ unit of time for all $T>0$.

Proof.

The sufficiency and the exact time controllability that follows are obvious.

For the converse assume the system to be controllable and let us denote by $\left(\mathcal{C}^{i} \mathfrak{g}\right)_{i \geq 1}$ the lower central series of $\mathfrak{g}$, that is $\mathcal{C}^{1} \mathfrak{g}=\mathfrak{g}$ and $\mathcal{C}^{i+1} \mathfrak{g}=\left[\mathfrak{g}, \mathcal{C}^{i} \mathfrak{g}\right]$ (see $[2])$.

Let $X$ be a right invariant vector field such that $\operatorname{ad}(X)=\operatorname{ad}(\mathcal{X})$, and let $k$ be the largest index such that $X \in \mathcal{C}^{k} \mathfrak{g}$. According to the definition of the lower central series the inclusion $\operatorname{ad}(X) \mathfrak{g} \subset \mathcal{C}^{k+1} \mathfrak{g}$ holds. On the other hand, the rank condition being satisfied, the smallest Lie subalgebra of $\mathfrak{g}$ that contains $\mathfrak{h}$ and is $\operatorname{ad}(X)$-invariant is equal to $\mathfrak{g}$.

Now $\mathfrak{h}+\mathcal{C}^{k+1} \mathfrak{g}$ is a subalgebra of $\mathfrak{g}$ (because $\mathcal{C}^{k+1} \mathfrak{g}$ is an ideal), and is $\operatorname{ad}(X)$-invariant, because $\operatorname{ad}(X)(\mathfrak{h}) \subset \operatorname{ad}(X)(\mathfrak{g}) \subset \mathcal{C}^{k+1} \mathfrak{g}$. This proves

$$
\mathfrak{h}+\mathcal{C}^{k+1} \mathfrak{g}=\mathfrak{g} .
$$

Let us assume that $\mathfrak{h}+\mathcal{C}^{k+r} \mathfrak{g}=\mathfrak{g}$ for some integer $r>0$. The field $X$ can be decomposed into $X=X_{h}+X_{r}$ where $X_{h} \in \mathfrak{h}$ and $X_{r} \in \mathcal{C}^{k+r} \mathfrak{g}$. Therefore

$$
\operatorname{ad}(X)(\mathfrak{h})=\operatorname{ad}\left(X_{h}\right)(\mathfrak{h})+\operatorname{ad}\left(X_{r}\right)(\mathfrak{h}) \subset \mathfrak{h}+\mathcal{C}^{k+r+1} \mathfrak{g} .
$$


This implies that $\mathfrak{h}+\mathcal{C}^{k+r+1} \mathfrak{g}$ is an $\operatorname{ad}(X)$-invariant subalgebra that contains $\mathfrak{h}$, hence equal to $\mathfrak{g}$.

We obtain by induction

$$
\forall r>0 \quad \mathfrak{h}+\mathcal{C}^{k+r} \mathfrak{g}=\mathfrak{g} .
$$

Since $\mathcal{C}^{k+r} \mathfrak{g}=\{0\}$ for $r$ large enough, the conclusion is $\mathfrak{h}=\mathfrak{g}$.

\section{Counterexamples}

1. Theorem 4 is no longer true whenever the derivation is not inner, as shown by the examples $L_{2}$ and $L_{4}$ on the group Heisenberg. Both systems are controllable, though the algebra $\mathfrak{h}$ has dimension 2 for $L_{2}$ and 1 for $L_{4}$.

2. This theorem is not true on solvable Lie groups either. Consider for instance the 2-dimensional non Abelian Lie algebra, $\mathfrak{g}=\operatorname{Vect}\{X, Y\}$ where $[X, Y]=Y$. It is a solvable Lie algebra whose all derivations are inner. Let $\mathfrak{h}=\mathbb{R} X$ and $\operatorname{ad}(\mathcal{X})=\operatorname{ad}(Y)$. Then $\mathfrak{h} \neq \mathfrak{g}$ but the algebra $L_{0}$ is equal to $\mathfrak{g}$ since $\operatorname{ad}(\mathcal{X}) X=\operatorname{ad}(Y) X=-Y$ (see also the example of Section 7.2).

\section{$6 \quad$ Affine vector fields}

In this section the drift of the system is an affine vector field $F$, that is the sum of a linear vector field $\mathcal{X}$ and a left invariant one $Z$. The system under consideration is:

$$
\left(\Sigma_{F}\right) \quad \dot{g}=F_{g}+\sum_{j=1}^{m} u_{j} Y_{g}^{j}
$$

Thanks to the decomposition $F=\mathcal{X}+Z$ the trajectories of $\Sigma_{F}$ can be compared with the ones of

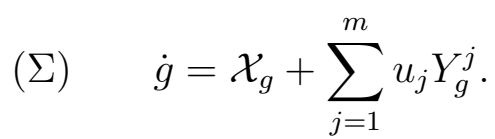

Proposition 10 The input $u$ being given, the solution of $\Sigma_{F}$ for the initial condition $g \in G$ is equal to $g(t) z(t)$ where 
1. $t \mapsto g(t)$ is the solution of $\Sigma$ that verifies $g(0)=g$;

2. $t \mapsto z(t)$ is the solution of $\dot{g}=\mathcal{X}_{g}+Z_{g}$ that verifies $z(0)=e$.

Proof. Let $\gamma(t)=g(t) z(t)$. According to Formula (1) of Section 2, we have for almost all $t \geq 0$ :

$$
\begin{aligned}
\frac{d}{d t} \gamma(t) & =T R_{z(t)}\left(\mathcal{X}_{g(t)}+\sum_{j=1}^{m} u_{j}(t) Y_{g(t)}^{j}\right)+T L_{g(t)}\left(\mathcal{X}_{z(t)}+Z_{z(t)}\right) \\
& =\mathcal{X}_{g(t) z(t)}+Z_{g(t) z(t)}+\sum_{j=1}^{m} u_{j}(t) Y_{g(t) z(t)}^{j} \\
& =\mathcal{X}_{\gamma(t)}+Z_{\gamma(t)}+\sum_{j=1}^{m} u_{j}(t) Y_{\gamma(t)}^{j} .
\end{aligned}
$$

Let us now assume the rank condition to be satisfied. Three cases have to be distinguished, and Proposition 10 will have a true interest only in the third one.

1. There exists $g_{0}$ such that $F\left(g_{0}\right)=0$. The rank condition requires $\mathcal{L}_{0}=\mathfrak{g}$, and by the Equivalence Theorem, recalled in Section 8.2, this case can be reduced to the one where the drift is linear. Actually the equivalence can be shown by applying to the system the right translation $R_{g_{0}^{-1}}$, denoted by $\Phi$ for abbreviation. For all $Y \in \mathfrak{g}$ we have $\Phi_{*} Y=Y$ and

$$
\left[\Phi_{*} F, Y\right]=\left[\Phi_{*} F, \Phi_{*} Y\right]=\Phi_{*}[F, Y]=[F, Y]
$$

since $[F, Y] \in \mathfrak{g}$. This proves that the field $\Phi_{*} F$ is affine, and henceforth linear since $\Phi_{*} F(e)=0$. Moreover $\operatorname{ad}\left(\Phi_{*} F\right)=\operatorname{ad}(F)=\operatorname{ad}(\mathcal{X})$ and $\Phi_{*} F$ is actually equal to $\mathcal{X}$.

Conclusion. The system is equivalent to the linear system $\dot{g}=\mathcal{X}(g)+\sum_{j=1}^{m} u_{j} Y_{g}^{j}$ where $\mathcal{X}=\Phi_{*} F$.

2. $\forall g \in G, F(g) \neq 0$ but $\mathcal{L}_{0} \neq \mathfrak{g}$. The rank condition implies:

- The codimension of $\mathcal{L}_{0}$ in $\mathfrak{g}$ is 1 .

- At the point $e$ we have $T_{e} G=\mathcal{L}_{0}(e) \oplus \mathbb{R} F(e)$. 
Let $\widetilde{G}$ be the connected and simply connected group whose Lie algebra is isomorphic to $\mathbb{R} F \oplus \mathcal{L}_{0}$. On account of the Equivalence Theorem, the group $G$ is diffeomorphic to the quotient of $\widetilde{G}$ by a subgroup $H$ (discrete because $G$ and $\widetilde{G}$ have the same dimension) and $\Sigma_{F}$ is equivalent to an invariant system on $G / H$. In the case where $G$ is simply connected, it is diffeomorphic to $\widetilde{G}$ and $\Sigma_{F}$ cannot be controllable: it is indeed a right invariant system whose vector fields are contained in a half-space bounded by the Lie subalgebra $\mathcal{L}_{0}$ (Hypersurface Principle, Cf [14]).

Furthermore Proposition 10 is of no help because the linear system $\Sigma$ does not satisfy the rank condition and is not controllable.

Conclusion. The system is equivalent to a right invariant one. It cannot be controllable if $G$ is simply connected.

3. $\forall g \in G, F(g) \neq 0$ and $\mathcal{L}_{0}=\mathfrak{g}$. In application of Proposition 10, and in a way similar to the one of Section 4.2 , the system $\Sigma_{F}$ is controllable as soon as $\Sigma$ is finite time controllable.

We can therefore state:

Proposition 11 With the previous notations it is assumed that the field $F$ does not vanish, and that the zero-time ideal $\mathcal{L}_{0}$ is equal to $\mathfrak{g}$. Then $\Sigma_{F}$ is finite time controllable if and only if $\Sigma$ is.

\subsection{Compact Lie groups}

Thanks to the analysis of the previous section, a general result of controllability on compact groups can be stated.

Theorem 5 The group $G$ is assumed to be compact.

System $\Sigma_{F}$ is controllable, hence finite time controllable, if and only if it satisfies the rank condition.

It is moreover exact time controllable if and only if $\mathcal{L}_{0}=\mathfrak{g}$. That condition is always satisfied in the case where the vector field $F$ is linear.

Proof.

1. Let us first prove the controllability statement in the case where $F=\mathcal{X}$ is linear. 
The group $G$ being compact its Lie algebra splits into $\mathfrak{g}=\mathfrak{z}+\mathfrak{s}$ where $\mathfrak{z}$ is the center of $\mathfrak{g}$ and $\mathfrak{s}$ is a semisimple compact ideal. The derivation associated to $\mathcal{X}$ is equal to $\operatorname{ad}(X)$ where $X$ can be choosen in $\mathfrak{s}$ (see [17] or [9]). The rank condition being satisfied, it is also satisfied by the invariant system associated to $\Sigma$ as in Section 4 . According to Theorems 7.1 and 7.2 of [10], this invariant system is finite time controllable, and on account of Proposition 9 the linear one is as well finite time controllable.

2. Let us now consider general drifts, and distinguish between the three cases. Thanks to the first item of this proof we obtain that $\Sigma_{F}$ is finite time controllable in the first case, and according to Proposition 11, in the third one. It is finite time controllable in the second case by virtue of the results of [10].

3. The last assertion is a consequence of a result of [8] recalled in Section 4.2 .

\section{Remarks.}

1. Controllability of linear systems on compact Lie groups has already been stated, with a different proof, in [17] (but nor the exact time controllability, neither the controllability of affine systems are proved in that paper).

2. Theorem 5 does not extend without additional assumptions to linear systems on compact homogeneous spaces: two counterexamples are exhibited in [9].

3. An exact time controllable linear system on a compact Lie group is not necessarily controllable in any time $t>0$. In the paper [10] an invariant system which is exact time controllable, but not in arbitrarily small time, is exhibited on $\mathrm{SO}_{3}$ (Example 8.1). The Lie algebra $\mathfrak{s o}_{3}$ being semisimple, the zero-time ideal of the system must be equal to $\mathfrak{s o}_{3}$. Consequently the linear system that can be associated satisfies also the rank condition. According to Proposition 9 this linear system is as well as the invariant one exact time controllable, but not in arbitrarily small time. 


\section{Examples}

\subsection{Examples on the Heisenberg group}

The Heisenberg group is the matrix group

$$
G=\left\{\left(\begin{array}{ccc}
1 & y & z \\
0 & 1 & x \\
0 & 0 & 1
\end{array}\right) ; \quad(x, y, z) \in \mathbb{R}\right\}
$$

Its Lie algebra $\mathfrak{g}$ is generated by the right invariant vector fields

$$
X=\left(\begin{array}{lll}
0 & 0 & 0 \\
0 & 0 & 1 \\
0 & 0 & 0
\end{array}\right), \quad Y=\left(\begin{array}{lll}
0 & 1 & x \\
0 & 0 & 0 \\
0 & 0 & 0
\end{array}\right), \quad Z=\left(\begin{array}{lll}
0 & 0 & 1 \\
0 & 0 & 0 \\
0 & 0 & 0
\end{array}\right),
$$

that verify $[X, Y]=Y X-X Y=Z$, and can be written in natural coordinates

$$
X=\frac{\partial}{\partial x}, \quad Y=\frac{\partial}{\partial y}+x \frac{\partial}{\partial z}, \quad Z=\frac{\partial}{\partial z} .
$$

\section{Example 1}

To the derivation $D$ of $\mathfrak{g}$ defined by $D X=Y, D Y=X$, and $D Z=0$ one associates the vector field $\mathcal{X}$ defined by

$$
\mathcal{X}=y \frac{\partial}{\partial x}+x \frac{\partial}{\partial y}+\frac{1}{2}\left(x^{2}+y^{2}\right) \frac{\partial}{\partial z} \text {. }
$$

A straightforward computation shows that this field is linear and verifies $-\operatorname{ad}(\mathcal{X})=D$. The system $\dot{g}=\mathcal{X}(g)+u X(g)$ can be written in $\mathbb{R}^{3}$

$$
\left(L_{1}\right)=\left\{\begin{array}{l}
\dot{x}=y+u \\
\dot{y}=x \\
\dot{z}=\frac{1}{2}\left(x^{2}+y^{2}\right)
\end{array}\right.
$$

Since $D X=Y$ and $[X, Y]=Z$, it satisfies the rank condition, but not the ad-rank one, because $D \mathfrak{g}=V \operatorname{Vect}\{X, Y\}$.

It is clearly not controllable on $G$, not even from the identity. It can be noticed that the equilibriums are the points $(0,0, z)$, with $u=0$, and that the linearized system is controllable at none of them. 
However the subgroup $H$ of $G$ defined by

$$
H=\left\{\left(\begin{array}{lll}
1 & 0 & n \\
0 & 1 & 0 \\
0 & 0 & 1
\end{array}\right) ; n \in \mathbb{Z}\right\}
$$

is discret and central. The linear field $\mathcal{X}$ can be projected to the quotient group $\widetilde{G}=G / H$, because $H$ is included in the set of fixed points of $\mathcal{X}$ (see [7]). The linear system thus defined on $\widetilde{G}=G / H$ is clearly controllable.

\section{Examples 2 and 3}

With the same linear field $\mathcal{X}$ as in Example 1, consider the systems:

$$
\left(L_{2}\right) \quad \dot{g}=\mathcal{X}(g)+u X(g)+v Z(g) \quad \text { and } \quad\left(L_{3}\right) \quad \dot{g}=\mathcal{X}(g)+u X(g)+v Y(g) \text {. }
$$

In natural coordinates, they write:

$$
\left(L_{2}\right)=\left\{\begin{array}{l}
\dot{x}=y+u \\
\dot{y}=x \\
\dot{z}=\frac{1}{2}\left(x^{2}+y^{2}\right)+v
\end{array} \quad \text { and } \quad\left(L_{3}\right)=\left\{\begin{array}{l}
\dot{x}=y+u \\
\dot{y}=x+v \\
\dot{z}=\frac{1}{2}\left(x^{2}+y^{2}\right)+v x
\end{array}\right.\right.
$$

These two systems are controllable on $G$. It is obvious for $L_{2}$, and the Lie saturate of $L_{3}$ contains $\pm X$ and $\pm Y$, hence also $\pm Z= \pm[X, Y]$.

Both systems satisfy the ad-rank condition. Indeed let us denote as previously by $\mathfrak{h}$ the subalgebra generated by the controlled fields. In the first case $\mathfrak{h}$ contains $X$ and $Z$, and also $Y=D X$. In the second one $\mathfrak{h}$ contains $X$ and $Y$, hence also $Z=[X, Y]$.

\section{Example 4}

In order to exhibit a single input system that satisfies the ad-rank condition, let us consider the derivation

$$
D=\left(\begin{array}{lll}
0 & 0 & 0 \\
1 & 0 & 0 \\
0 & 1 & 0
\end{array}\right)
$$

The linear vector field associated to this derivation is

$$
\mathcal{X}=x \frac{\partial}{\partial y}+\left(y+\frac{1}{2} x^{2}\right) \frac{\partial}{\partial z}
$$

The system $\dot{g}=\mathcal{X}(g)+u X(g)$ can be written in coordinates

$$
\left(L_{4}\right)=\left\{\begin{array}{l}
\dot{x}=u \\
\dot{y}=x \\
\dot{z}=y+\frac{1}{2} x^{2}
\end{array}\right.
$$


Let us denote by $\mathcal{Z}$ the center of $G$, that is the closed subgroup $\{x=y=0\}$. As in Example 1 the linear field $\mathcal{X}$ can be projected to the quotient group $\widetilde{G}=G / \mathcal{Z}$, because $\mathcal{Z}$ is included in the set of fixed points of $\mathcal{X}([7])$. The induced system on $G / \mathcal{Z}$ is equivalent to the linear, in the usual meaning, system on $\mathbb{R}^{2}$ :

$$
\left\{\begin{array}{l}
\dot{x}=u \\
\dot{y}=x
\end{array}\right.
$$

which is clearly controllable. On the other hand $\mathcal{Z}$ is included in $\mathcal{A}$. Indeed the linearized system of $L_{4}$ at the identity is controllable, and in particular the set $\mathcal{A}_{t} \cap \mathcal{Z}$ is a neighbourood of $e$ in $\mathcal{Z}$ for all $t>0$. As the restriction of $\varphi_{t}$ to $\mathcal{Z}$ is the identity, the set $\mathcal{A} \cap \mathcal{Z}$ is a semigroup: if $g \in \mathcal{A}_{t} \cap \mathcal{Z}$ and $g^{\prime} \in \mathcal{A}_{s} \cap \mathcal{Z}$, then

$$
g g^{\prime}=g \varphi_{t}\left(g^{\prime}\right) \in \mathcal{A}_{t+s} \cap \mathcal{Z} .
$$

Consequently $\mathcal{A} \cap \mathcal{Z}=\mathcal{Z}$. Taking into account the controllability on the quotient, it is easy to see that the system is controllable from the identity. In a similar way it is controllable to the identity, hence controllable.

\subsection{An example on the group $A f f_{+}(2)$}

Let $G$ be the connected component of $e$ in the 2-dimensional affine group:

$$
G=A f f_{+}(2)=\left\{\left(\begin{array}{ll}
x & y \\
0 & 1
\end{array}\right) ; \quad(x, y) \in \mathbb{R}_{+}^{*} \times \mathbb{R}\right\}
$$

Its Lie algebra $\mathfrak{g}$ is identified with the set of left invariant vector fields. This 2-dimensional algebra is solvable and generated by

$$
A=\left(\begin{array}{ll}
1 & 0 \\
0 & 0
\end{array}\right) \quad \text { and } \quad B=\left(\begin{array}{ll}
0 & 1 \\
0 & 0
\end{array}\right)
$$

with $[A, B]=A B-B A=B$. The matrices $A$ and $B$ are identified with the left invariant vector fields:

$$
g A=\left(\begin{array}{ll}
x & 0 \\
0 & 0
\end{array}\right) \quad \text { and } \quad g B=\left(\begin{array}{ll}
0 & x \\
0 & 0
\end{array}\right) \quad \text { where } \quad g=\left(\begin{array}{ll}
x & y \\
0 & 1
\end{array}\right) .
$$

Let $\mathcal{X}$ be the linear field defined by $\mathcal{X}(g)=g B-B g=\left(\begin{array}{cc}0 & x-1 \\ 0 & 0\end{array}\right)$, and consider the linear system $\Sigma_{L}: \dot{g}=\mathcal{X}(g)+u g A$ and the left invariant one $\Sigma_{I}: \dot{g}=g B+u g A$. 
The linear system $\Sigma_{L}$ satisfies the rank condition, because $D A=[A, B]=$ $B$, hence both systems satisfy the rank condition. In natural coordinates they write:

$$
\left(\Sigma_{L}\right)=\left\{\begin{array}{l}
\dot{x}=u x \\
\dot{y}=x-1
\end{array} \quad \text { and } \quad\left(\Sigma_{I}\right)=\left\{\begin{array}{l}
\dot{x}=u x \\
\dot{y}=x
\end{array} \quad \text { for }(x, y) \in \mathbb{R}_{+}^{*} \times \mathbb{R} .\right.\right.
$$

The invariant system is not controllable: indeed $x$ is positive, and for any input $y(t) \geq y(0), \forall t \geq 0$.

Let us show that the linear system is controllable, and for this purpose let us consider $\Sigma_{L}$ as a polysystem. The initial point $\left(x_{i}, y_{i}\right)$ can be steered to the final one $\left(x_{f}, y_{f}\right)$ in three steps:

1. Following the field $\pm g A$ the point $\left(x_{i}, y_{i}\right)$ can be steered to $\left(\frac{1}{2}, y_{i}\right)$ if $y_{f}<y_{i}$, or to $\left(\frac{3}{2}, y_{i}\right)$ if $y_{f}>y_{i}$, in arbitrarily small time;

2. the constant control $u=0$ steers the point $\left(\frac{1}{2}, y_{i}\right)\left(\right.$ resp. $\left.\left(\frac{3}{2}, y_{i}\right)\right)$ to the point $\left(\frac{1}{2}, y_{f}\right)\left(\operatorname{resp} .\left(\frac{3}{2}, y_{f}\right)\right)$ in time $2\left|y_{f}-y_{i}\right|$.

3. The field $\pm g A$ leads to the final point.

\subsection{Examples on the group $S L_{2}$}

\section{Example 1}

Consider the matrices $A$ and $B$ of $\mathfrak{s l}_{2}$ defined by

$$
A=\left(\begin{array}{cc}
a & 1 \\
-1 & -a
\end{array}\right) \quad \text { and } \quad B=\left(\begin{array}{cc}
-1 & 0 \\
0 & 1
\end{array}\right)
$$

The right invariant system $\Sigma_{I}$ and the linear one $\Sigma_{L}$ are defined by:

$$
\begin{array}{ll}
\left(\Sigma_{I}\right) & \dot{g}=A g+u B g \\
\left(\Sigma_{L}\right) & \dot{g}=A g-g A+u B g
\end{array}
$$

For all $a \in \mathbb{R}$ the invariant system is controllable on $S L_{2}$ (see [15] for the detailed proof). 
In order to analyse the controllability of the linear system we set $\mathcal{X}(g)=$ $A g-g A, D=-\operatorname{ad}(\mathcal{X})=-\operatorname{ad}(A)$, and we obtain (for the bracket $[B, A]=$ $A B-B A)$

$$
D B=\left(\begin{array}{ll}
0 & 2 \\
2 & 0
\end{array}\right) \quad \text { et } \quad D^{2} B=-4 B+a\left(\begin{array}{cc}
0 & 1 \\
-1 & 0
\end{array}\right) .
$$

- If $a \neq 0$ the rank of $\left\{B, D B, D^{2} B\right\}$ is equal to 3 so that the ad-rank condition is satisfied. The invariant system being controllable, Theorem 1 applies, and $\Sigma_{L}$ is controllable.

- If $a=0$, the ad-rank condition is not satisfied. However the one parameter group

$$
\exp (t A)=\left(\begin{array}{cc}
\cos t & \sin t \\
-\sin t & \cos t
\end{array}\right)
$$

is periodic, and the flow $\varphi_{t}(g)=\exp (t A) g \exp (-t A)$ is as well periodic, hence Poisson stable. The linear system is therefore controllable.

The case where $a=0$ is an example of a controllable system that does not satisfy the ad-rank condition. Notice however that the group $S L_{2}$ is not simply connected.

\section{Example 2}

This example can be found in [17].

Consider the matrices $X$ and $Y$ of $\mathfrak{s l}_{2}$ defined by

$$
X=\left(\begin{array}{cc}
1 & 0 \\
0 & -1
\end{array}\right) \quad \text { and } \quad Y=\left(\begin{array}{cc}
1 & 1 \\
1 & -1
\end{array}\right)
$$

They verify

$$
[X, Y]=\left(\begin{array}{cc}
0 & -2 \\
2 & 0
\end{array}\right) \quad \text { and } \quad[X,[X, Y]]=\left(\begin{array}{ll}
0 & 4 \\
4 & 0
\end{array}\right)
$$

hence $\left\{Y, D Y, D^{2} Y\right\}$, where $D=-\operatorname{ad}(X)$, is a basis of $\mathfrak{s l}_{2}$. Henceforth the linear system

$$
\left(\Sigma_{I}\right) \quad \dot{g}=X g-g X+u Y g
$$

satisfies the ad-rank condition, and is locally controllable from the identity in $S L_{2}$. 
It is however not controllable, not even from $e$. Indeed if it was, then the invariant system

$$
\left(\Sigma_{I}\right) \quad \dot{g}=X g+u Y g
$$

would also be controllable in account of Theorem 2. In that case the bilinear system $\dot{x}=X x+u Y x$ induced by $\Sigma_{I}$ in $\mathbb{R}^{2}$ would be controllable on $\mathbb{R}^{2} \backslash\{0\}$. But that system has invariant orthants: the eigenspaces of $Y$, generated by the vectors $(1, \sqrt{2}-1)$ and $(1-\sqrt{2}, 1)$, define four orthants in $\mathbb{R}^{2}$. The one that contains the point $(1,0)$ is positively invariant for the bilinear system which is therefore not controllable on $\mathbb{R}^{2} \backslash\{0\}$.

\section{Appendix}

\subsection{The theorem of [5]}

The theorem of El Assoudi-Gauthier-Kupka deals with semisimple Lie groups with finite center. Its statement requires some definitions. The complexification of the algebra $\mathfrak{g}$ of the group is denoted by $\mathfrak{g}_{\mathbb{C}}$, and the adjoint representation of $\mathfrak{g}_{\mathbb{C}}$ by ad $\mathfrak{C}_{\mathbb{C}}$. The real part of a complex number $z$ is denoted by $\Re(z)$, and its imaginary part by $\Im(z)$.

Definition. An element $Y \in \mathfrak{g}$ is said to be strongly regular if:

(i) $Y$ is a regular element of $\mathfrak{g}$.

(ii) All the non-vanishing, real or complex, eigenvalues of ad $(Y)$ are simple.

Let us denote by $\operatorname{Sp}(Y)$ the set of non-vanishing, real or complex, eigenvalues of $\operatorname{ad}(Y)$. For $a \in \operatorname{Sp}(Y)$, let $\mathfrak{g}_{a}$ stand for the eigenspace of $\mathfrak{g}_{\mathbb{C}}$ associated to $a$ (it is 1 -dimensional). The algebra $\mathfrak{g}_{\mathbb{C}}$ splits into:

$$
\mathfrak{g}_{\mathbb{C}}=\operatorname{ker}\left(\operatorname{ad}_{\mathbb{C}}(Y)\right) \oplus\left(\oplus_{a \in \operatorname{Sp}(Y)} \mathfrak{g}_{a}\right)
$$

and the corresponding decomposition of $Z \in \mathfrak{g}_{\mathbb{C}}$ is denoted by:

$$
Z=Z_{0}+\sum_{a \in \operatorname{Sp}(Y)} Z_{a}
$$


Definition Let us endow $\mathbb{C}$ with the lexicographic order: $a<b$ if $\Re(a)<\Re(b)$ or if $\Re(a)=\Re(b)$ and $\Im(a)<\Im(b)$.

An eigenvalue $a \in S p(Y)$ is said to be maximal (resp. minimal), if for all $b \in S p(Y)$ with $0<b($ resp. $b<0)$

$$
\left[\mathfrak{g}_{a}, \mathfrak{g}_{b}\right]=\{0\} .
$$

The theorem can know be stated:

\section{Theorem (El Assoudi, Gauthier, Kupka)}

Let $G$ be a real, connected, semisimple Lie group with finite center, the Lie algebra of which is denoted by $\mathfrak{g}$. The system

$$
\dot{g}=X_{g}+\sum_{j=1}^{m} u_{j} Y_{g}^{j}
$$

where $X, Y^{1}, \ldots, Y^{m} \in \mathfrak{g}$, is controllable as soon as the following conditions are satisfied:

(H1) The vector fields $X, Y^{1}, \ldots, Y^{m}$ generate the Lie algebra $\mathfrak{g}$.

(H2) There exists in the Lie subalgebra $\mathfrak{h}$ generated by $Y^{1}, \ldots, Y^{m}$ a strongly regular element $Y$.

(H3) Let $X=X_{0}+\sum_{a \in S p(Y)} X_{a}$ be the decomposition of $X$ along the eigenspaces of $a d_{\mathbb{C}}(Y)$. Then $X_{a} \neq 0$ if the eigenvalue $a \in S p(Y)$ is minimal or maximal.

(H4) If $a \in S p(Y)$ is maximal, if its real part $r=\Re(a)$ is a non-vanishing eigenvalue of ad $d_{\mathbb{C}}(Y)$, and if $\mathfrak{g}_{r}$ and $\mathfrak{g}_{a}$ belong to the same simple ideal of $\mathfrak{g}_{\mathbb{C}}$, then the trace of ad $\left(X_{r}\right) \circ \operatorname{ad}\left(X_{-r}\right)$ is negative.

\subsection{The Equivalence Theorem}

Let us recall that an affine vector field $F$ is obtained by adding a left invariant vector field $Z$ to a linear one $\mathcal{X}$. It may be more suitable to see $F$ as the sum of a linear vector field and a right invariant one. This can be done by writing:

$$
F=\mathcal{X}+Z=\mathcal{X}+Z+\mathcal{I}_{*} Z-\mathcal{I}_{*} Z=\widetilde{\mathcal{X}}-\mathcal{I}_{*} Z
$$


where $\tilde{\mathcal{X}}=\mathcal{X}+Z+\mathcal{I}_{*} Z$ is linear (recall that $\mathcal{I}$ is defined by $\mathcal{I}(g)=g^{-1}$ ).

Let $H$ be a closed subgroup of $G$, and let $F=\mathcal{X}+Y$, where $Y$ is right invariant, be an affine vector field. The projection of $F$ onto the homogeneous space $G / H$ (manifold of left cosets of $H$ ) exists if and only if the subgroup $H$ is $\mathcal{X}$-invariant. In that case it will be refered to as an affine vector field on the homogeneous space $G / H$ (see [7] for the characterization of such vector fields).

The definition of a linear system is generalized in the following way: a linear system on a Lie group or a homogeneous space is defined as

$$
\dot{x}=F(x)+\sum_{j=1}^{m} u_{j} Y_{j}(x)
$$

where $F$ is an affine vector field and the $Y_{j}$ 's are right invariant if the state space is a Lie group, and projections of right invariant vector fields if the state space is a homogeneous space.

Let us consider the following smooth system, defined on a connected manifold $M$ :

$$
\text { (S) } \quad \dot{x}=f(x)+\sum_{j=1}^{m} u_{j} g_{j}(x)
$$

\section{Equivalence Theorem [7]}

We assume the family $\left\{f, g_{1}, \ldots, g_{m}\right\}$ to be transitive. Then System $S$ is diffeomorphic to a linear system on a Lie group or a homogeneous space if and only if the vector fields $f, g_{1}, \ldots, g_{m}$ are complete and generate a finite dimensional Lie algebra.

More accurately, let $G$ (resp. $G_{0}$ ) be the connected and simply connected Lie group whose Lie algebra is $\mathcal{L}$ (resp. $\left.\mathcal{L}_{0}\right)$. Under the previous conditions the rank of $\mathcal{L}_{0}$ is constant, equal to $\operatorname{dim}(M)$ or $\operatorname{dim}(M)-1$, and:

(i) if rang $\left(\mathcal{L}_{0}\right)=\operatorname{dim}(M)$, in particular if there exists one point $p_{0} \in M$ such that $f\left(p_{0}\right)=0$, then $S$ is diffeomorphic to a linear system on a homogeneous space $G_{0} / H$ of $G_{0}$;

(ii) if rang $\left(\mathcal{L}_{0}\right)=\operatorname{dim}(M)-1$, then $S$ is diffeomorphic to an invariant system on a homogeneous space $G / H$ of $G$. 


\section{References}

[1] V. Ayala and J. Tirao Linear control systems on Lie groups and Controllability, Proceedings of Symposia in Pure Mathematics, Vol 64, AMS, 1999, 47-64.

[2] N. Bourbaki Groupes et algèbres de Lie, Chapitre 1, CCLS, 1972.

[3] N. Bourbaki Groupes et algèbres de Lie, Chapitres 2 et 3, CCLS, 1972.

[4] F. Cardetti and D. Mittenhuber Local controllability for linear control systems on Lie groups, Journal of Dynamical and Control Systems, Vol. 11, No. 3, July 2005, 353-373.

[5] R. El Assoudi, J.P. Gauthier, I. Kupka On subsemigroups of semisimple Lie groups, Ann. Inst. Henri Poincaré, Vol. 13, No 1, 117-133 (1996).

[6] Ph. Jouan On the existence of observable linear systems on Lie Groups, Journal of Dynamical and Control Systems, Vol. 15, No 2, April 2009, 263-276.

[7] Ph. Jouan Equivalence of Control Systems with Linear Systems on Lie Groups and Homogeneous Spaces ESAIM: Control Optimization and Calculus of Variations, 16 (2010) 956-973.

[8] Ph. Jouan Finite Time and Exact Time Controllability on Compact Manifolds to appear in the Journal of Mathematical Sciences.

[9] Ph. Jouan Invariant measures and controllability of finite systems on compact manifolds, submitted.

[10] V. Jurdjevic and H.J. Sussmann Control Systems on Lie Groups, Journal of Differential Equations 12, 313-329 (1972).

[11] V. Jurdjevic Geometric control theory, Cambridge university press, 1997.

[12] L. Markus Controllability of multitrajectories on Lie groups, Dynamical systems and turbulence, Warwick 1980, pp. 250-265, LN in Math., 898, Springer, Berlin-New York, 1981. 
[13] H. Nijmeijer and A.J. van der Schaft Nonlinear Dynamical Control Systems, Springer 1990.

[14] Yu.L. Sachkov Controllability of Invariant Systems on Lie Groups and Homogeneous Spaces, Journal of Mathematical Sciences, Vol. 100, No. 4, 2000 .

[15] Yu.L. Sachkov Control Theory on Lie groups, Journal of Mathematical Sciences, Vol. 156, No. 3, 2009.

[16] L. San Martin Nonreversibility of Subsemigoups of Semi-Simple Lie Groups Semigroup Forum Vol. 44 (1992) 376-387.

[17] L. San Martin and V. Ayala Controllability properties of a class of control systems on Lie Groups Nonlinear control in the year 2000, Vol. 1 (Paris), 83-92, Lecture Notes in Control and Inform. Sci., 258, Springer, London, 2001.

[18] L. San Martin and P. Tonelli Semigroup actions on homogeneous spaces, Semigroup Forum Vol. 50 (1995) 59-88.

[19] E.D. Sontag Mathematical Control Theory, second edition, Springer, New-York, 1998. 